\title{
Uptake of first two doses of human papillomavirus vaccine by adolescent schoolgirls in Manchester: prospective cohort study
}

\author{
Loretta Brabin, reader in women's health, ${ }^{1}$ Stephen A Roberts, senior lecturer in statistics, ${ }^{2}$ \\ Rebecca Stretch, research nurse, ${ }^{1}$ David Baxter, consultant in communicable diseases, ${ }^{3}$ Gloria Chambers, \\ programme manager screening failsafe, ${ }^{4}$ Henry Kitchener, professor of gynaecological oncology, ${ }^{1}$ \\ Rosemary McCann, consultant in communicable diseases ${ }^{5}$
}

\begin{abstract}
Academic Unit of Obstetrics and Gynaecology, St Mary's Hospital, University of Manchester

Manchester M13 OJH

${ }^{2}$ Health Methodology Research Group, University of Manchester

${ }^{3}$ Public Health Department, Stockport Primary Care Trust, Stockport

${ }^{4}$ Public Health Department, Bury Primary Care Trust, Bury

${ }^{5}$ Greater Manchester Health

Protection Unit, Eccles

Correspondence to: L Brabin
\end{abstract}

loretta.brabin@manchester.ac.uk

doi:10.1136/bmj.39541.534109.BE

\section{ABSTRACT}

Objective To assess the feasibility and acceptability of delivering a human papillomavirus (HPV) vaccine to adolescent girls.

Design Prospective cohort study.

Setting 36 secondary schools in two primary care trusts in Greater Manchester, United Kingdom.

Participants 2817 schoolgirls in year 8 (12 and 13 year olds).

Intervention Delivery of the bivalent vaccine at 0,1 , and 6 months over one school year.

Main outcome measures Vaccine uptake for doses 1 and 2 of a three dose schedule.

Results Vaccine uptake was $70.6 \%(1989 / 2817)$ for the first dose and $68.5 \%$ (1930/2817) for the second dose. Uptake was significantly lower in schools with a higher proportion of ethnic minority girls ( $\mathrm{P}<0.001$ for trend) or higher proportion of girls entitled to free school meals $(P=0.029$ for trend). The main reason for parents' refusal of vaccination was insufficient information about the vaccine and its long term safety. Maintaining the vaccine schedule was challenging as $16.3 \%$ (dose 1) and $23.6 \%$ (dose 2) of girls missed theirvaccination day and had to be offered alternative appointments. No serious adverse events were reported.

Conclusion Delivery of the first two doses of HPV vaccine to adolescent schoolgirls is encouraging, but the success of the vaccination programme depends on high coverage for the third dose.

\section{INTRODUCTION}

From September 2008, schoolgirls aged 12 or 13 years (year 8) in the United Kingdom will routinely be offered vaccination with one of two licensed vaccines against human papillomavirus (HPV) — a quadrivalent vaccine (Gardasil; Merck, PA, USA) or a bivalent vaccine (Cervarix; GlaxoSmithKline, Rixensart, Belgium). ${ }^{1}$ In uninfected females both vaccines effectively prevent HPV-16 and HPV-18 associated cervical intraepithelial neoplasia, which is responsible for about $70 \%$ of cervical cancers. ${ }^{2}$ Two studies on parents' attitudes to the vaccines in the UK have predicted an uptake of $70-80 \%,{ }^{34}$ but generalised acceptability is not certain because no precedent exists for routine delivery of a vaccine against a sexually transmitted infection to this age group. ${ }^{5}$

The effectiveness of the national immunisation programme depends on good coverage. ${ }^{6} \mathrm{We}$ assessed the feasibility of delivering vaccination against HPV to adolescent girls and the acceptability of vaccination to parents. We report on uptake of the first and second doses of the bivalent vaccine among girls attending schools in two primary care trusts in Greater Manchester.

\section{METHODS}

In February 2007 we invited all 10 primary care trusts in Greater Manchester to join the study. Two agreed. Each primary care trust was responsible for delivering the vaccine to all secondary schools in its catchment area. Each developed a plan to implement delivery of the vaccine at 0,1 , and 6 months and a process for reporting serious adverse events. Parents were fully informed about the study. They received a flier summarising the content of an educational film for girls, ${ }^{7}$ details of parents' evenings, a slip to indicate reasons for refusal, and a separate consent for a followup research questionnaire. Parents were sent letters and reminders by post, with a prepaid envelope for reply. Information evenings were organised in both primary care trusts. Each offered rescheduled visits for missed appointments.

The child's details were transferred to a single proforma recording the three vaccine doses. Child health departments of the two trusts informed general practitioners of the child's vaccination status and collated anonymised data. Local education authorities supplied aggregated data on the characteristics of the girls, including school types, ethnic composition, and entitlement to free school meals. The denominator for calculating uptake was the number of girls offered vaccination. We used logistic regression to explore the 
Table 1| Number and proportion of 12 and 13 year old schoolgirls receiving first two doses of three dose vaccination schedule against human papillomavirus

\begin{tabular}{lc} 
Vaccination status & No (\%) of schoolgirls $(\mathbf{n}=\mathbf{2 8 1 7})$ \\
Unvaccinated: & $824(29.4)$ \\
\hline No response to invitation & $571(20.3)$ \\
\hline Refused vaccination & $228(8.1)$ \\
\hline Consented to vaccination & $29(1.0)$ \\
\hline Received first dose: & $1989(70.6)$ \\
\hline On schedule & $1665(59.1)$ \\
\hline Later* & $324(11.5) \dagger$ \\
\hline Received second dose: & $1930(68.5)$ \\
\hline On schedule & $1474(52.3)$ \\
\hline Later* & $456(16.2) \ddagger$ \\
\hline Missed second dose & $59(2.1) \S$ \\
\hline
\end{tabular}

*Vaccinated either at later visit to school or at community follow-up clinic.

$\dagger 16.3 \%$ of girls vaccinated.

$\$ 23.6 \%$ of girls vaccinated.

$\$ 3.0 \%$ of girls receiving first dose.

relation between characteristics of the school (as continuous variables) and uptake, excluding two schools that did not fully participate.

\section{RESULTS}

HPV vaccine was offered to 2817 year 8 girls attending 36 secondary schools. In total, $1989(70.6 \%)$ received the first vaccine dose and $29(1.0 \%)$ remained unvaccinated (table 1). Among the vaccinated group alternative sessions had to be arranged for girls who missed their first appointment and for late consenters (16.3\% of those vaccinated). Uptake for the second dose was $68.5 \%(1930 / 2817)$. Almost a quarter of these girls were vaccinated at times other than the initially scheduled visit because they were absent, were vaccinated at an interval longer than six weeks after

Table 2 | Parents' reasons for not consenting to vaccination of their daughters against human papillomavirus

Reason for refusal

No $(\%)$ of parents stating reason* $(n=148)$

Information:

\begin{tabular}{lc}
\hline Insufficient to make a decision, or many unknowns & $54(36)$ \\
\hline Safety, especially long term & $47(32)$ \\
\hline Efficacy (for example, need for booster) & $9(6)$ \\
\hline Context: & $19(13)$ \\
\hline Study or research & $15(10)$ \\
\hline Waiting for national programme & $24(16)$ \\
\hline Would prefer quadrivalent vaccine & $15(10)$ \\
\hline Timing of vaccine: & $11(7)$ \\
\hline Young age & $4(3)$ \\
\hline Child is low risk & \\
\hline Message vaccine gives: & $6(4)$ \\
\hline Condones sexual activity & $4(3)$ \\
\hline Contraindications: & $11(7)$ \\
\hline Individual practicalities (for example, moving home) & $4(3)$ \\
\hline Medical contraindication & \\
\hline Perceived contraindication (for example, vaccine phobia) \\
\hline Other (for example, improve sex education instead)
\end{tabular}

the first dose $(92,3.3 \%)$, or were vaccinated at less than 28 days after the first dose $(49,1.7 \%)$. No serious adverse events were recorded. Vaccine uptake was significantly lower in schools with a higher proportion of girls from ethnic minority groups $(\mathrm{P}<0.001$ for trend $)$ or girls with entitlement to free school meals $(\mathrm{P}=0.029$ for trend).

Among the unvaccinated group, $571(20.3 \%)$ parents did not reply to the invitation letter and $228(8.1 \%)$ returned a refusal form. Overall, 148 (65\%) parents who refused gave at least one reason for not providing consent (table 2). The main reason was insufficient information about the vaccine and its long term safety. Ten per cent of those parents who refused consent did not want to participate in a research study, preferred to wait for the national programme, or preferred the quadrivalent vaccine. Few parents mentioned the age for vaccination of their daughters or the vaccine's effect on adolescent sexual behaviour.

\section{DISCUSSION}

It is possible to deliver and achieve an acceptable level of coverage for the first two doses of a three dose vaccination schedule against HPV in schoolgirls aged 12 and 13 years. Delivery is challenging because these doses need to be delivered at the start of the academic year when schools are busy. The interval between doses is short and up to a quarter of girls did not attend their scheduled appointment. About 3\% of girls missed the second dose; it will be important to maintain coverage for the third dose.

Although the vaccination programme was designed to follow routine implementation, this was a research study. This context led to a reduced uptake with some parents refusing vaccination stating that they were waiting for the national programme. The differences from routine practice were the additional consent to allow contact by the research team, a request to return the consent form even if the vaccine was refused, and the information sheet explaining the nature of the study. Taking this into account, a coverage of $80 \%$, anticipated by several studies on acceptability of the vaccine, ${ }^{8}$ may be achievable. We are unsure why $20 \%$ of parents did not respond to the invitation. Sociodemographic factors may be important, ${ }^{910}$ but a reliable association between vaccine uptake and sociodemographic characteristics would require a larger study.

To date little published data are available on HPV coverage from countries where the vaccine has been introduced - in Ontario, Canada, a first dose uptake of $53 \%$ has been quoted. ${ }^{11}$ A comparable feasibility study in the UK for vaccination of adolescents against hepatitis B virus showed a coverage of $91 \%$ for the first dose, decreasing to $80 \%$ for the third dose. ${ }^{12}$

The main reason for parents refusing initial consent was lack of familiarity with the vaccine and worries about as yet unrecognised adverse events. Two schools refused to participate on religious grounds, although three other schools of the same religious denomination did take part. These are encouraging results for the 


\section{WHAT IS ALREADY KNOWN ON THIS TOPIC}

Countries that vaccinate adolescent girls against HPV have not yet published data on coverage

UK studies anticipate about $80 \%$ uptake but no acceptability or feasibility studies have been done

\section{WHAT THIS STUDY ADDS}

Two primary care trusts offering HPV vaccination to girls attending all secondary schools in the area achieved a $70 \%$ uptake for the first vaccine dose

The vaccine was acceptable to most parents, and school based delivery was feasible

forthcoming national HPV vaccine programme but the final criterion for success will be the proportion of girls who receive all three vaccine doses.

We thank staff of the primary care trust and school for working against a tight timescale to bring this project to fruition and the education departments of the two councils for providing school census data. Contributors: LB conceived the study and wrote the paper. She is guarantor. SAR designed the study and analysed the data. RS managed the study, collected the data, and contributed to the analysis; DB and GC implemented the vaccination programme. HK commented on the analysis and revisions to the paper. RMcC facilitated the research, chaired the steering committee, and commented on the paper.

Funding: This study was sponsored by the University of Manchester. Vaccine delivery was the responsibility of the primary care trusts. GlaxoSmithKline funded the research and implementation costs and provided the vaccine. The authors' work was independent of the funders, who played no part in the conduct of the research or vaccine delivery. LB is funded by the Max Elstein Foundation.

Competing interests: $L B$ and $H K$ have received research funds, conference fees, and honorariums for speaking at meetings sponsored by GlaxoSmithKline.
Ethical approval: This study was approved by the north Manchester National Health Service research ethics committee.

Provenance and peer review: Not commissioned; externally peer reviewed.

1 Department of Health. HPV vaccine recommended for NHS immunisation programme. www.gnn.gov.uk/environment (accessed 26 Oct 2007).

2 Rambout L, Hopkins L, Hutton B, Fergusson D. Prophylactic vaccination against human papillomavirus infection and disease in women: a systematic review of randomized controlled trials. CMAJ 2007;177:469-79.

3 Brabin L, Roberts RA, Farzaneh F, Kitchener HC. Future acceptance of adolescent human papillomavirus vaccination: a survey of parental attitudes. Vaccine 2006;24:3087-94.

4 Marlow LAV, Waller J, Wardle J. Parental attitudes to pre-pubertal HPV vaccination. Vaccine 2007;25:1945-52.

5 Raffle AE. Challenges of implementing human papillomavirus (HPV) vaccination policy. BMJ 2007;335:375-7.

6 Kohli M, Ferko N, Martin A, Franco EL, Jenkins D, Gallivan S, et al. Estimating the long-term impact of a prophylactic human papillomavirus $16 / 18$ vaccine on the burden of cervical cancer in the UK. Br I Cancer 2007;96:143-50.

7 Vallely LA, Roberts S, Kitchener H, Brabin L. Informing adolescents about HPV vaccination. What will parents allow? Vaccine 2008, doi:10.1016/j.vaccine.2008.02.055.

8 Zimet GD, Liddon M, Rosenthal SL, Lazcano-Ponce E, Allen B. Psychosocial aspects of vaccine acceptability. Vaccine 2006;24(suppl 3):201-9.

9 Middleton E, Baker D. Comparison of social distribution of immunisation with measles, mumps and rubella vaccine, England, 1991-2001. BMJ 2003;326:854.

10 Mixer RE, Jamrozik K, Newsom D. Ethnicity as a correlate of the uptake of the first dose of mumps, measles and rubella vaccine. J Epidemiol Community Health 2007;61:797-801.

11 Breakthrough vaccine victim of bad publicity. Canadian National Post. 19 Feb,

2008. www.nationalpost.com/news/canada/Story.html? id $=317535$.

12 Wallace LA, Bramley JC, Ahmed S, Duff R, Hutchinson SJ, Carman WF, et al. Determinants of universal hepatitis B vaccine uptake. Arch Dis Child 2004:89:1041-2.

Accepted: 1 April 2008 